\title{
Shock probes in a one-dimensional Katz-Lebowitz-Spohn model
}

\author{
Sakuntala Chatterjee ${ }^{1,2}$ and Mustansir Barma ${ }^{1}$ \\ ${ }^{1}$ Department of Theoretical Physics, Tata Institute of Fundamental Research, Homi Bhabha Road, Mumbai-400005, India \\ ${ }^{2}$ Institut für Festkörperforschung, Forschungzentrum Jülich, D-52425 Jülich, Germany
}

(Received 27 November 2007; published 17 June 2008)

\begin{abstract}
We consider shock probes in a one-dimensional driven diffusive medium with nearest-neighbor Ising interaction (KLS model). Earlier studies based on an approximate mapping of the present system to an effective zero-range process concluded that the exponents characterizing the decays of several static and dynamical correlation functions of the probes depend continuously on the strength of the Ising interaction. On the contrary, our numerical simulations indicate that over a substantial range of the interaction strength, these exponents remain constant and their values are the same as in the case of no interaction (when the medium executes an ASEP). We demonstrate this by numerical studies of several dynamical correlation functions for two probes and also for a macroscopic number of probes. Our results are consistent with the expectation that the short-ranged correlations induced by the Ising interaction should not affect the large time and large distance properties of the system, implying that scaling forms remain the same as in the medium with no interactions present.
\end{abstract}

DOI: 10.1103/PhysRevE.77.061124

PACS number(s): 02.50.Ey, 05.40.-a, 64.75.Gh, 05.60.-k

\section{INTRODUCTION}

Useful information about a complex system is often obtained by introducing probe particles into it. After the probe particles have come to a steady state with the system, their static and dynamic behavior often reflect important characteristics of the system. For instance, by monitoring the motion of probe particles, one can understand viscoelastic properties of a cell [1], the sol-gel transition in a polymer solution [2] or correlations present in bacterial motion [3]. In certain cases, for example, in active microrheology, the probe particles are subjected to external force fields. In Ref. [4] the forced dynamics of a magnetic bead in a dense colloidal suspension, has been used to study the colloidal glass transition. In this paper we will consider one such example of nonequilibrium (driven) probe particles which are introduced in a nonequilibrium medium, to study how the static and dynamic properties of the probe particles are influenced by the surrounding medium, and also how the medium gets affected by the presence of the probe particles.

We study a particular simple one-dimensional lattice gas model first introduced in Ref. [5] to describe the motion of probe particles in a current-carrying medium. The probe particles are taken to exchange with particles and holes of the medium with equal rates but in opposite directions. Because of these dynamical rules, the probe particles tend to migrate toward the region of strong density variations (or shocks) which may be present in the system. Studying the dynamics of these shock-tracking probe particles, one can therefore infer the motion of density fluctuations in the medium.

In an earlier study [6], we have discussed the dynamical properties of these probe particles in a nonequilibrium current-carrying medium in which there is no interaction between medium particles except hard-core exclusion. In this case, the medium was described by an asymmetric simple exclusion process (ASEP) which is the simplest lattice model of driven diffusive systems [7]. The shock-tracking probe particles then reduce to second class particles [8]. Derrida et al. have found the exact stationary measure of the system [9]. Their studies on static properties of the system show that when the number of second class particles is finite, they form a bound state and the steady state distribution function of the separation $r$ between a pair decays as $r^{-\lambda}$ with $\lambda=3 / 2$. A macroscopic number of second class particles gives rise to a correlation length which diverges (proportional to the square of the interprobe separation), as the probe concentration goes to zero.

We studied the dynamical properties of this system in presence of a macroscopic number of probe particles, and found that the dynamics is governed by a time scale which marks the crossover from single-probe behavior to manyprobe behavior [6]. This time scale shows a strong divergence (proportional to the cube of the interprobe separation) in the limit of vanishingly small density of the probe particles. This diverging time scale is related to the diverging correlation length present in the system [9], and enters the scaling descriptions of various dynamical correlation functions of the probe particles [6].

In the present paper, we present a detailed study of shocktracking probes in a driven system in which there is a shortranged Ising interaction between the particles of the medium. In the absence of any probes, such a medium can be described by the one-dimensional (1D) Katz-Lebowitz-Spohn (KLS) model, whose steady state has an Ising measure $[10,11]$. In [5] Kafri et al. have reported that in presence of a macroscopic number of probe particles, the system shows an interesting phase transition as the strength of the Ising interaction is varied. Beyond a critical value of the interaction strength and for sufficiently high density of the medium, a macroscopic domain consisting of particles and holes (no probes) is formed. A characterization of this phase transition was attempted using an approximate mapping to the zerorange process (ZRP) where the probes are mapped onto ZRP sites and the particle-hole domain preceding a probe is mapped onto the occupancy of that site [5]. The current out of a particle-hole domain then becomes the hopping rate out of a site in the ZRP. 
A prediction of this approximate mapping is that exponents characterizing the decays of several static and dynamic quantities should depend continuously on the Ising interaction strength $\epsilon$. However, our numerical studies of these quantities seem to indicate that the exponents are $\epsilon$ independent over a substantial range of $\epsilon$. This paper is concerned with a study of the differences between our results and those based on the ZRP picture.

A possible simple rationalization of our results is that the Ising interactions would be expected to give rise to a finite correlation length $\xi_{\text {Ising }}$, whose value may be renormalized in the presence of probes, but is still expected to be finite. Then, on length scales $r \gg \xi_{\text {Ising }}$, the system should behave essentially as the noninteracting $(\epsilon=0)$ system. Our results are indeed consistent with such a scenario, as we find $\epsilon$-independent behavior asymptotically (for large $r$ and $t$ ), even though there is sometimes an $\epsilon$ dependence for smaller $r$ and $t$. Below we describe in brief the quantities we studied and our results.

(1) Distribution function of the size of the particle-hole domains. The mapping to the ZRP predicts that in the disordered phase this distribution function should be an exponential times a power law, with a power which is a continuous function of the Ising interaction strength. However, we observe that the domain size distribution shows a power law exponent which does not vary with $\epsilon$ but remains constant at its value for $\epsilon=0$. To understand this discrepancy, we are led to check the assumptions that have been made in the approximate KLS-ZRP mapping. We find that the assumption of statistical independence of the domains remains valid, and further verify that accounting for the finite size correction to domain currents is not the reason behind the discrepancy. However, as we discuss in Sec. III, the movement of probes in a KLS medium is non-Markovian and the ZRP mapping does not capture this aspect of probe dynamics.

(2) Dynamics of two probe particles. This was studied in Refs. $[12,13]$ where it was reported that a bound state forms between the probe pair such that the distribution of separation decays as a power law with an exponent $b(\epsilon)$ that varies continuously with the strength of the Ising interaction $\epsilon$. Starting from a configuration where the two probes were nearest neighbors, a scaling form was proposed to describe the temporal evolution of their separation. The authors have tried to verify this scaling form by measuring the cumulative distribution and the mean value of the separation between the probes as a function of time. They reported that in conformity with their scaling hypothesis, the time-dependent cumulative distribution function $\widetilde{P}(r, t)$ for different values of $t$ undergoes a scaling collapse when rescaled by $t^{[b(\epsilon)-1] / z}$ and plotted against $r t^{-1 / z}$, where $z$ is the dynamical exponent that takes the value $3 / 2$. The average separation between the two probes is reported to grow with time as a power law with an exponent $[2-b(\epsilon)] / z$, which is consistent with their scaling form.

On the contrary, we find that although for an initial time range, the average distance does show $\epsilon$-dependent growth, for larger times, it crosses over to another growth regime where the exponent takes a value which is close to the one expected for $\epsilon=0$, i.e., with no Ising interaction. Our numeri- cal results also show that for larger times, the scaling collapse of $\widetilde{P}(r, t)$ fails. We have verified that the scaling collapse can be retrieved by rescaling with $t^{1 / 3}$ (instead of $\left.t^{[b(\epsilon)-1] / z}\right)$, as in the case of $\epsilon=0$.

(3) Dynamical properties with a macroscopic number of probes. The dynamical correlation functions in this case are found to follow the same scaling description as with $\epsilon=0$, with a crossover time scale which separates a single-probe regime at short times from a long-time regime characterized by collective behavior of the probes. Moreover, the crossover time scale shows a similar divergence in the limit of vanishingly low concentration of the probe particles. In other words, our studies indicate that even in the presence of a nearest-neighbor Ising interaction in the medium, the large time and large distance properties of the system do not change.

In the following section, we describe the lattice model on which we have performed Monte Carlo simulation and briefly summarize our earlier results for the noninteracting $(\epsilon=0)$ medium. In Sec. III we discuss the static properties of this model where we recall the approximate mapping to the zero-range process (ZRP) introduced in Ref. [5] and discuss the validity of various assumptions that went into this mapping. In Sec. IV we discuss the dynamical properties of the system in presence of a finite number of probes and also for a finite density of the probes.

\section{MODEL AND EARLIER RESULTS $(\epsilon=0)$}

The model is defined on a one dimensional periodic lattice each site of which may either be empty or may contain a particle of the medium or a probe. We use the symbol "+" to denote a particle, "-" to denote a hole, and " 0 " to denote a probe. The exchange rules are as follows:

$$
+-\stackrel{1-\Delta V}{\longrightarrow}-+, \quad+0 \stackrel{1}{\longrightarrow} 0+, \quad 0-\stackrel{1}{\rightarrow}-0 .
$$

Here $\Delta V$ is the change in the nearest-neighbor Ising interaction potential

$$
V=-\frac{\epsilon}{4} \sum_{i} s_{i} s_{i+1},
$$

where $s_{i}=0, \pm 1$, according to whether the site $i$ contains a probe, a particle, or a hole, respectively. Throughout we consider equal densities of particles and holes in the medium, i.e., $\rho_{0}=1-2 \rho$, where $\rho$ and $\rho_{0}$ denote densities of particles and probes, respectively. The coupling parameter $\epsilon$ may vary in the range $[-1,1]$. In this paper, we will only consider $\epsilon$ $>0$.

In the absence of any probes, the system reduces to a 1D KLS model with an Ising measure in the steady state. This gives rise to an $\epsilon$-dependent correlation length $\xi_{\text {Ising }}$ in the system. For $\epsilon<1$, this correlation length remains finite and hence the large distance properties of the system can be expected to remain unaffected by the interaction.

When probes are present, as seen from the last two exchange rules in Eq. (1), a probe exchanges with particles and holes of the medium in opposite directions. This implies that 
a probe would tend to be located in a position where there is an excess of holes to its left and an excess of particles to its right. In other words, there would be a strong density variation or "shock" around a probe. This is the reason we call them "shock-tracking probes" (STPs).

In the absence of any interaction, one has $\epsilon=0$ and in this case, a particle in the medium executes a totally asymmetric exclusion process (TASEP) with an effective hole density $(1-\rho)$ and it exchanges with a hole and a probe in the same way; similarly, a hole in the medium also executes a TASEP [in the opposite direction and with an effective particle density $\left.\left(\rho+\rho_{0}\right)\right]$ and it exchanges with a particle and a probe in the same way. In other words, for $\epsilon=0$ a probe behaves like a particle for an adjacent hole and like a hole for an adjacent particle. Such probes are known as "second class particles" [8].

Derrida et al. [9] have found the exact steady state measure of this system of second class particles in an ASEP by using the matrix method. In presence of more than one second class particle, the steady state factorizes about any second class particle, which implies factorization in terms of the one component system about the shock position. When there is a single second class particle present in the system, the mean density profile around it decays as a power law with an exponent $1 / 2$. In the presence of two (or a finite number of) second class particles, the medium induces an attraction between them and they form a weakly bound state where the distance $r$ between two successive second class particles follows a power law distribution $P(r) \sim r^{-3 / 2}$. When the number of second class particles is macroscopic, the density profile at a distance $r$ from any given probe takes the form

$$
\rho(r) \sim \frac{1}{\sqrt{r}} \exp (-r / \xi)+\rho,
$$

where the correlation length $\xi$ diverges in the low concentration limit of the probes [9]:

$$
\xi \approx 4 \rho(1-\rho) / \rho_{0}^{2} \text { as } \rho_{0} \rightarrow 0 .
$$

We monitored several quantities to study the dynamical properties of systems with macroscopic number of probes. We find a diverging time scale which marks the crossover between single-probe behavior and many-probe behavior. In Sec. IV, we will discuss the behavior of these quantities, when $\epsilon$ is nonzero in the KLS model.

The variance of the displacement of the tagged probes is defined as

$$
C_{0}(t)=\left\langle\left[Y_{k}(t)-Y_{k}(0)-\left\langle Y_{k}(t)-Y_{k}(0)\right\rangle\right]^{2}\right\rangle
$$

where $Y_{k}(t)$ is the position of the $k$ th probe at time $t$. Ferrari and Fontes [14] had earlier calculated the asymptotic $(t \rightarrow \infty)$ behavior of $C_{0}(t)$ and shown that $C_{0}(t) \approx D t$ with diffusion constant $D=\left[\rho(1-\rho)+\left(\rho+\rho_{0}\right)\left(1-\rho-\rho_{0}\right)\right] / \rho_{0}$. For small times, in the limit of low concentration of the probe particles, one would expect each probe to behave as an individual noninteracting particle subject only to the fluctuations of the medium. The variance of the displacement of a single probe is found analytically to grow as $t^{4 / 3}[15,16]$. In the limit of small but finite concentration of the probe particles
$C_{0}(t)$ shows a single-particle (superdiffusive) behavior at small time and diffusive behavior at asymptotically large times. One would therefore expect a crossover between these two regimes that would occur at a time scale $\tau$ which is a function of $\rho_{0}$. The natural expectation would be $\tau \sim \xi^{2}$ where $\xi$ is the correlation length as defined in Eq. (4). Substituting the value of the dynamical exponent $z=3 / 2$ and using Eq. (4) one obtains

$$
\tau \sim \rho_{0}^{-3}
$$

in the limit of small $\rho_{0}$. This leads us to propose the following scaling form for $C_{0}(t)$ :

$$
C_{0}(t) \sim t^{4 / 3} F\left(\frac{t}{\tau}\right) .
$$

This form is valid in the scaling limit of large $t$ and large crossover time scale $\tau$ (i.e., $\rho_{0} \rightarrow 0$ ). Here $F(y)$ is a scaling function which approaches a constant as $y \rightarrow 0$. For $y \gg 1$, we must have $F(y) \sim y^{-1 / 3}$, in order to reproduce $C_{0}(t) \approx D t$. We have verified the above scaling form by Monte Carlo simulation [6].

The same crossover time scale $\tau$ is found to be present in other dynamical correlation functions as well. To track the dissipation of the density pattern of the second class particles, we considered the quantity

$$
B_{0}(t)=\overline{\left\{Y_{k}(t)-Y_{k}(0)-\overline{\left[Y_{k}(t)-Y_{k}(0)\right]}\right\}^{2}},
$$

where the overhead bar denotes averaging over different evolution histories, starting from a fixed initial configuration drawn from the steady state ensemble (see Refs. $[17,18]$ and also Ref. [6] for a discussion on why this special averaging process is useful in tracking dissipation). Our scaling analysis leads to the following scaling form

$$
B_{0}(t) \sim t^{4 / 3} G\left(\frac{t}{\tau}\right),
$$

where $\tau$ is the same crossover time scale as in Eq. (7) and $G(y)$ is a scaling function which approaches a constant as $y \rightarrow 0$, while for $y \gg 1$, one expects $G(y) \sim y^{-2 / 3}$. Our numerical results are consistent with this scaling form [6].

Finally consider the quantity

$$
\Delta(t)=\left\langle[R(t)-R(0)]^{2}\right\rangle
$$

which measures how the separation between two successive probes fluctuates in time. Here, $R(t)$ is the separation between the $k$ th and $(k+1)$-th pair at time $t$. Our studies show that $\Delta(t)$ has the following scaling form:

$$
\Delta(t) \sim t H\left(\frac{t}{\tau}\right),
$$

where the scaling function $H(y)$ approaches a constant as $y$ $\rightarrow 0$ and for $y \gg 1$ one must have $H(y) \sim 1 / y$.

To summarize, for $\epsilon=0$ we find that several dynamical correlation functions of the probe particles are governed by a single crossover time scale $\tau$ which diverges as $\rho_{0}^{-3}$ for low concentration of the probes. In the remaining portion of the 


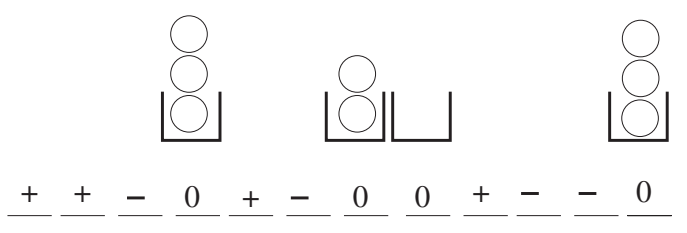

FIG. 1. A typical configuration of the KLS model with probes and its corresponding configuration in ZRP.

paper, we will consider static and dynamical properties for $\epsilon>0$ and examine how different they are from the noninteracting case.

\section{STATIC PROPERTIES OF KLS MODEL WITH PROBES}

Kafri et al. reported that the KLS model with macroscopic number of probes shows phase separation transition for $\epsilon$ $>0.8$ as the density $\rho$ is increased above a critical value $\rho_{c}$ [5]. They concluded that in the phase separated state, a macroscopic domain, composed of particles and holes of the medium, coexists with another phase which consists of small domains of particles and holes, separated by the probes. They explained this phase transition by attempting to approximately map the system onto a zero-range process.

To describe the mapping, we first define a domain as an uninterrupted sequence of particles and holes, bounded by probes from both ends. The current $J_{n}$ out of a domain of length $n$ can then be determined by studying a KLS model in an open chain with boundary rates of injection and extraction equal to the rate at which the particles and holes of the domain would exchange with the probes at the domain boundaries. According to Eq. (1) this rate is unity. The current $J_{n}$ can be calculated exactly for an open KLS chain and for large $n$ it has the form

$$
J_{n}=J_{\infty}\left(1+\frac{b(\epsilon)}{n}\right),
$$

where the coefficient $b$ has the following dependence on $\epsilon$ :

$$
b(\epsilon)=\frac{3}{2} \frac{(2+\epsilon) v+2 \epsilon}{2(v+\epsilon)}, \quad v=\sqrt{\frac{1+\epsilon}{1-\epsilon}}+1 .
$$

The study of Kafri et $a l$. indicates that $b$ plays an important role in characterizing the phase separation transition in the model.

The present system is mapped onto a zero-range process (ZRP) as follows: the $i$ th probe is defined as the $i$ th site of ZRP and the length of the domain to the left of the $i$ th probe is taken to be the occupancy $n(i)$ of the $i$ th site of ZRP. We illustrate this in Fig. 1. The density in the ZRP is related to the KLS model density $\rho$ as $\rho_{\mathrm{ZRP}}=2 \rho /(1-2 \rho)$.

The hopping rate out of the $i$ th site in the ZRP is taken to be the domain current $J_{n(i)}$ given in Eq. (12). For such a ZRP, the condition for condensation to take place is $b>2$ and $\rho_{\mathrm{ZRP}}$ larger than a certain critical density $\rho_{c}$. In the condensed phase, the occupancy at a single site becomes macroscopically large, while the remaining sites have an average occupancy $\rho_{c}$ [19]. For $\rho_{\mathrm{ZRP}}<\rho_{c}$, the number of particles present on a site follows the distribution function

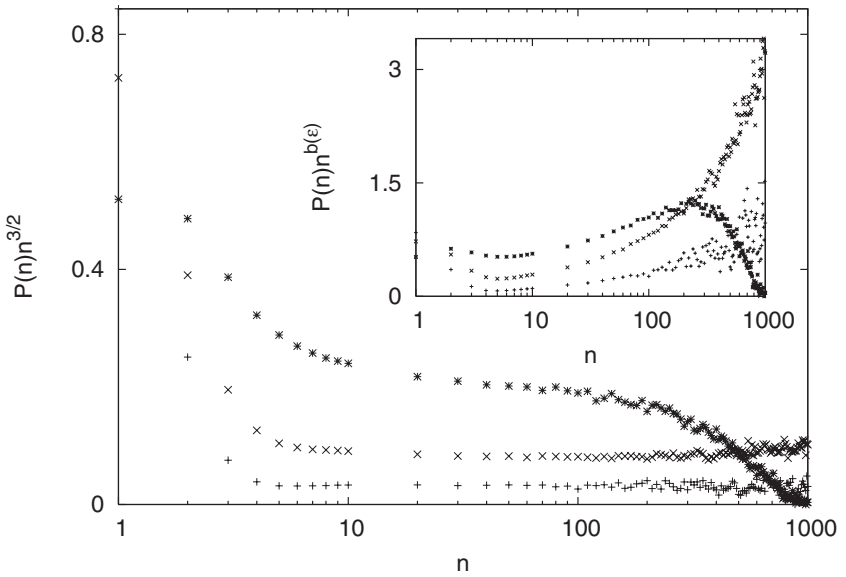

FIG. 2. Domain size distribution $P(n)$ scaled by $n^{3 / 2}$ shows a flat stretch over a substantial range of $n$ for different values of $\epsilon$ and $\rho$. For comparison with the ZRP prediction, we have scaled $P(n)$ by $n^{b(\epsilon)}$ in the inset. In both these plots, the symbol + corresponds to $\epsilon=0.9, \rho=0.464$, symbol $\times$ corresponds to $\epsilon=0.8, \rho=0.375$ and symbol * corresponds to $\epsilon=0.6, \rho=0.375$. In the last case, $\epsilon$ is substantially smaller than the critical value and $\xi_{\text {ZRP }}$ is shorter. This explains the observed deviation from the power law behavior for large $n$.

$$
P(n) \sim \frac{1}{n^{b}} \exp \left(-n / \xi_{\text {ZRP }}\right)
$$

where the correlation length $\xi_{\mathrm{ZRP}}$ diverges as $\rho_{\mathrm{ZRP}} \rightarrow \rho_{c}$. For $\rho_{\mathrm{ZRP}}=\rho_{c}$, we have $P(n) \sim 1 / n^{b}$, while for $\rho_{\mathrm{ZRP}}>\rho_{c}$, a similar power law decay describes the distribution at all sites except for the single condensate site.

The approximate ZRP correspondence implies that in the KLS chain with probes, for large enough $\rho$ and for $\epsilon>0.8$ [as follows from Eq. (13)], there should be a macroscopic domain present in the system which is composed of particles and holes (no probes). The rest of the system should consist of small probe clusters, interrupted by the domains (of particles and hole) with size distribution given by Eq. (14).

From numerical simulations for $\epsilon<0.8$, it was found that a very large domain may exist for large $\rho$ [5]. Our numerical simulations confirm this. In Refs. $[5,20]$ it has been argued that this is not true phase separation, but rather a consequence of the fact that the correlation length in this case has a large (but finite) value.

According to the above correspondence with the ZRP, it is expected that close to the critical point, the domain size distribution for $n \ll \xi_{\text {ZRP }}$ should follow a power law with exponent $b(\epsilon)$ which should increase monotonically with $\epsilon$. However, our numerical simulations for various values of $\epsilon$ and $\rho$ (see Fig. 2) show that the power law exponent seems throughout to be close to $3 / 2$ (which is the value of $b$ at $\epsilon$ $=0)$, independent of the value of $\epsilon$. This points to a contradiction with the correspondence with the ZRP, and leads us to examine the assumptions that go into the KLS-ZRP mapping.

Independence of domains. A crucial property of the ZRP is that the occupancies at the sites are uncorrelated. In our present model of the KLS chain with probes, this would 


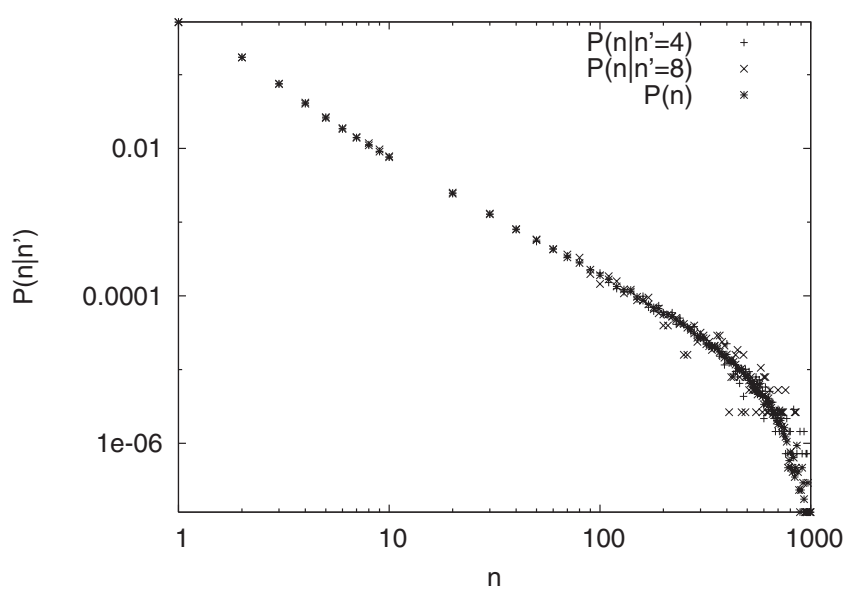

FIG. 3. The conditional distribution of domain size $P\left(n \mid n^{\prime}\right)$ as a function of $n$ for $n^{\prime}=4,8$. For comparison $P(n)$ is also shown. $P\left(n \mid n^{\prime}\right)$ is seen to match with $P(n)$ which shows the domains are independently distributed. We have used $L=2048, \epsilon=0.6$, and $\rho$ $=0.375$.

imply that the domains between the probes should be independently distributed. We have verified this assumption by measuring the conditional probability $P\left(n \mid n^{\prime}\right)$ that the size of a particular domain is of length $n$ given that its neighboring domain is of length $n^{\prime}$. We find that $P\left(n \mid n^{\prime}\right)$ does not depend on $n^{\prime}$ and is same as $P(n)$ consistent with neighboring domains being distributed independently. Our data is presented in Fig. 3.

Finite size correction to domain current. Apart from the independence of domains, another requirement for the ZRP mapping to hold is that the current out of a domain of size $n$ should be the same as the current in an isolated open KLS chain and is given by Eq. (12). Evans et al. have given evidence for this by numerically measuring the actual current out of a domain and comparing with the exact calculation for an open chain KLS model [21]. Good agreement was found for large $n$.

To take into account the finite size corrections for moderate values of $n$, we simulated a ZRP where the hopping rate out of a site is read off directly from the actual $J_{n}$ vs $n$ data, obtained from numerical simulation of the KLS model with probes. The mass distribution for this ZRP is found to have the same form as in Eq. (15) with the exponent $b$ given by Eq. (14), as expected. We conclude that the finite size correction to $J_{n}$ is not the reason for the discrepancy shown in Fig. 2.

Non-Markovian movement of the probes. There is, however, one aspect of the KLS model with probes that is not captured in the corresponding ZRP. Since a probe exchanges with the particles and holes of the medium in opposite directions, as shown in Eq. (1), once a probe moves in one particular direction, it cannot move in the opposite direction at the very next time step. For example, suppose a probe moves to the left by exchanging with a particle in the medium. Immediately after this exchange the probe has the particle as its right neighbor. Clearly, the probe cannot take a step to the right as long as that particle stays there. In other words, the probes have a finite memory which makes their movement
non-Markovian. In terms of the ZRP this would mean that once a site has emitted a particle to its right neighbor, it has to wait for some time till it can receive a particle from its right neighbor. This waiting time should depend on the form of the density profile in a domain. Note that in this nonMarkovian ZRP, apart from $J_{\infty}$ and $b(\epsilon)$, there are other parameters that are associated with the exact form of the waiting time. As a result, the phase diagram becomes complicated and to specify the criterion of a phase transition a much more detailed analysis is required. This might shed some light on the observed discrepancy about domain size distribution.

\section{DYNAMICS OF PROBES IN THE KLS MODEL}

\section{A. Two probes}

The properties of two STPs in the KLS chain were first studied in Ref. [12] by Levine et al., who argued that the time evolution of the separation between the probe pair is governed by a Master equation. Their analysis indicates that the medium induces an attraction among the probe particles and they form a bound state. The steady state distribution of the distance between two probes takes the form $P(r) \sim r^{-b}$ where $b$ is a function of $\epsilon$ given by Eq. (13). For $\epsilon=0$ one retrieves $P(r) \sim r^{-3 / 2}$ as found in Ref. [9].

Rakos et al. have shown that the random force between the probe pair is sensitive to the noise correlations present in the medium [13]. When the probe particles are embedded in a KLS ring, such that the random force that drives the probe particles is fully generated by the current fluctuations of the driven medium, the probes inherit the dynamical exponent of the medium, which is $3 / 2$. On the other hand, if the random force has a part that is temporally uncorrelated, the resulting motion is described by a dynamical exponent $z=2$.

To study the dynamics of the system, the distance between the two probes was monitored, starting from the initial configuration in which the two probes were side by side. The approach to the steady state was modeled by the scaling ansatz

$$
P(r, t) \sim r^{-b} f\left(r / t^{1 / z}\right),
$$

where $P(r, t)$ is the probability that starting as nearest neighbors, the two probes are at a distance $r$ apart at time $t$. In the range $1<b<2$ this would imply that the average distance between the two probes grows as

$$
\langle r(t)\rangle \sim t^{(2-b) / z}
$$

Since $b$ is an increasing function of the Ising interaction $\epsilon$, this would predict a slower growth law of $\langle r\rangle$ with $t$, as $\epsilon$ increases.

The cumulative distribution function $\widetilde{P}(r, t)$ is defined as the probability that starting from a nearest-neighbor position, the separation between the two probes at time $t$ is larger than $r$. From Eq. (15) it follows that

$$
\widetilde{P}(r, t) \sim t^{(1-b) / z} Y\left(r / t^{1 / z}\right),
$$

which means that $\widetilde{P}(r, t) t^{(b-1) / z}$, plotted against $r / t^{1 / z}$ should show a scaling collapse for various values of $t$. 


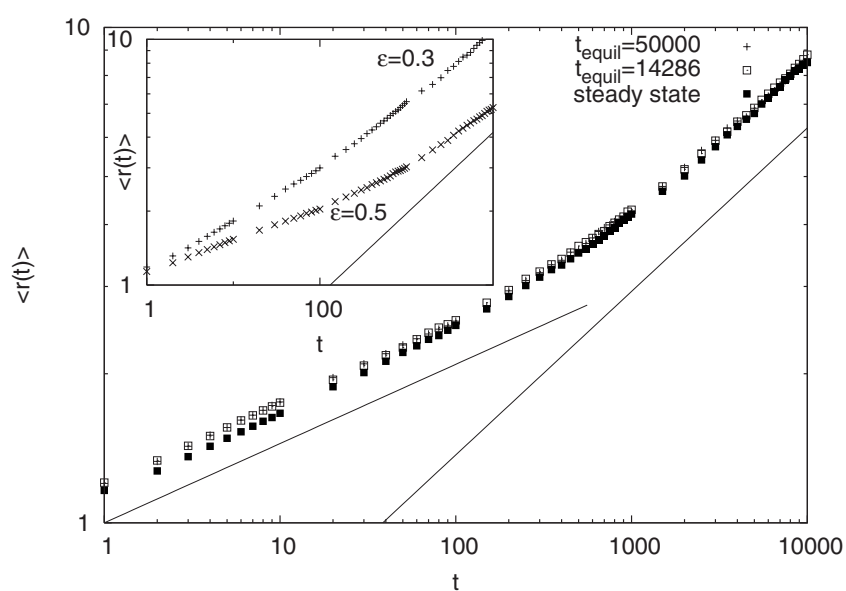

FIG. 4. Average distance $\langle r(t)\rangle$ between the probe pair as a function of time. $\langle r(t)\rangle$ shows two different power law growths as time changes. The reference lines show that the growth exponent is $(2-b) / z$ at short times and changes to $1 / 3$ at large times. The curves for partially equilibrated initial conditions (using the method of Rakos et al.) with different values of $t_{\text {equil }}$ coincide for small $t$. We have also measured $\langle r(t)\rangle$ starting from steady state initial condition. The partially equilibrated data and steady state data coincide for large $t$. We have used $\epsilon=0.4$ and $L=1000$. The inset shows the steady state data for $\epsilon=0.3,0.5$ with $L=4096$ and the reference line with exponent $1 / 3$.

In Refs. $[12,13]$ the time evolution of the average distance between the two probes was monitored numerically. Starting from a randomly disordered configuration, with the restriction that the two probes are placed on nearest neighbor sites, the system was evolved for a time $t_{\text {equil }}$ in an attempt to let it reach an equilibrium state. The time evolution during the equilibration process followed the exchange rules shown in Eq. (1) with the important modification that the two probes were constrained to remain nearest neighbors, i.e., they hopped together as if glued together. At the end of this equilibration, the medium is assumed to be locally in steady state, in the vicinity of the probes, up to a distance of the order $t_{\text {equil }}^{2 / 3}$. At this point, defined as $t=0$, the restriction for the relative position of the probes was released and the distance between them monitored. The distance between the probes was then assumed to follow the scaling form in Eq. (15) for $t \ll t_{\text {equil }}$ when the two probes move within an equilibrated region. In this time regime, it was numerically verified that the growth of $\langle r(t)\rangle$ is consistent with Eq. (16) $[12,13]$.

Note that the scaling form in Eq. (15) is expected to be valid in steady state. Therefore, to verify this scaling form, we followed the following procedure. Allow the system to reach steady state by evolving it without any restriction on the relative separation of the two probes. Then wait till the probes come to a nearest-neighbor position with respect to each other and define $t=0$ at this point. Our data shows that $\langle r(t)\rangle$ follows Eq. (16) only for an initial time-regime, after which the growth exponent changes to $\simeq 1 / 3$ which is close to the value of the growth exponent at $\epsilon=0$. We present our data in Fig. 4.

We have also measured $\langle r(t)\rangle$ following the procedure of Refs. [12,13]. We investigated the effect of different values of $t_{\text {equil }}$ and found the same behavior as described in the last
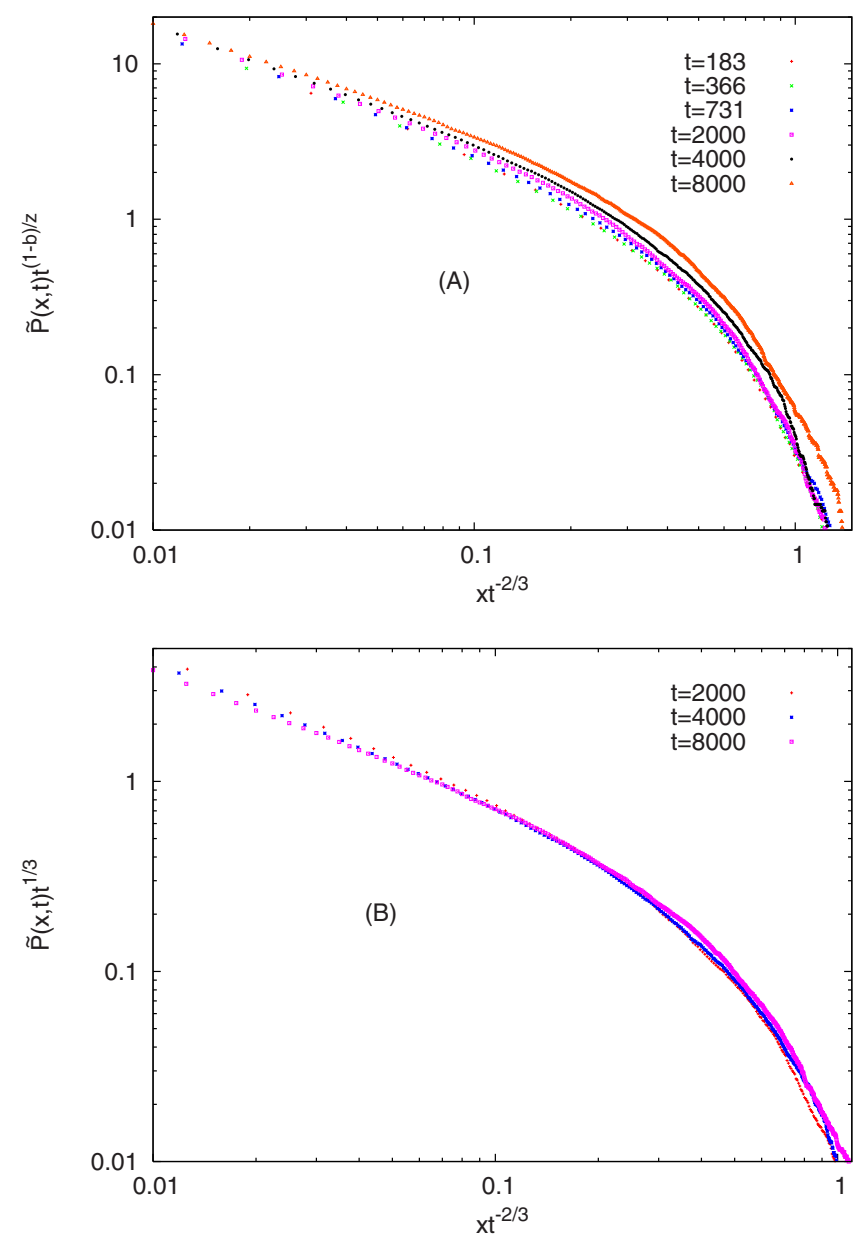

FIG. 5. (Color online) (A) shows the lack of scaling collpse for the cumulative distribution function $\widetilde{P}(x, t)$ for larger values of $t$. As seen from the label of $y$ axis, when $\widetilde{P}(x, t)$ is rescaled by an $\epsilon$-dependent prefactor, the scaling collapse works for smaller $t$, but fails for larger $t$.This is in contradiction with the scaling form described in Eq. (17). Instead, $\widetilde{P}(x, t)$ is seen to follow a scaling form $\widetilde{P}(x, t) \sim t^{-1 / 3} X\left(x / t^{2 / 3}\right)$, for larger values of $t$, as shown in panel (B). We have used $L=4096$ and $\epsilon=0.4$.

paragraph. Moreover, Fig. 4 shows that the curves for this partially equilibrated initial condition, coincide with that of the steady state initial condition (explained in the previous paragraph), for large time. We conclude that in steady state, $\langle r(t)\rangle$ does not follow Eq. (16) with an $\epsilon$-dependent $b$ all the way, but shows a crossover at large time to the behavior $t^{1 / 3}$, which is the behavior obtained for $\epsilon=0$.

In Ref. [13] it was also reported that the cumulative distribution function $\widetilde{P}(r, t)$ shows a scaling form as in Eq. (17). Starting from an initial configuration with the two probes next to each other (as discussed above), $\widetilde{P}(r, t)$ was numerically measured for a range of values of $t$ and it was concluded that within that range, $\widetilde{P}(r, t) t^{2(b-1) / 3}$ shows a scaling collapse for different values of $t$, as plotted against $r / t^{3 / 2}$. However, our numerical results indicate that this scaling collapse fails for larger $t$ values [see Fig. 5(a)].

Instead, an $\epsilon$-independent scaling form, more specifically, the scaling form expected for $\epsilon=0$, seems to hold. We show 
this by plotting $\widetilde{P}(r, t) t^{1 / 3}$ against $r / t^{3 / 2}$ and Fig. 5(b) shows the scaling collapse for larger $t$ values.

In our simulation, we could not go to very large times as the finite size effects would become strong. In Fig. 5 we have presented our data for the largest system size $(L=4096)$ we could access. However, the crossover time is much smaller and no finite size effects are observed for this time range.

Our studies therefore show that the large time dynamics of the two probes is not affected by the presence of an interaction in the medium as reported in Refs. [12,13], rather it resembles the case of non-interating medium. As discussed in Sec. I, one possible rationalization is that the Ising measure of a KLS model (without probes) induces a finite $\epsilon$-dependent correlation length in the medium. In the presence of probes, the value of this correlation length may change, but it is expected to be finite still. As long as the displacement of the probes is less than this correlation length, the effect of varying $\epsilon$ may be felt. But asymptotically, when the typical probe separation has exceeded the Ising correlation length, it is plausible that they behave as if in a medium with no interactions, i.e., $\epsilon=0$ [22].

\section{B. Macroscopic number of probes}

We now take up the study of a system with a macroscopic number of probes. We find that the dynamics of the STPs is governed by a diverging time-scale $\tau$, as in the noninteracting case $\epsilon=0$. For $t \ll \tau$, an STP senses the fluctuations solely due to the KLS chain. But a KLS chain is known to have an Ising measure which means that if $\epsilon$ is not too close to unity, only short-ranged correlations are present in the medium. Let $\tau_{0}$ be the time required for a probe particle to move a distance of order $\xi_{\text {Ising. }}$. Then for $\tau_{0} \ll t \ll \tau$, the dynamics of the probes in a KLS chain should be similar to those in an ASEP (where no correlation is present in the medium), i.e., as that of the second class particles discussed in Ref. [6]. The dependence of the crossover time $\tau$ on the probe density is discussed below.

Let $r_{i}$ be the separation between the $i$ th and $(i+1)$-th probe and $R_{m}$ be the distance between the first and the ( $m$ $+1)$-th probe, i.e., $R_{m}=\sum_{i=1}^{m} r_{i}$. Let $r_{i}$ follow the distribution $P\left(r_{i}\right) \sim r_{i}^{-\lambda}$. Assuming independence, the quantity $R_{m}$ which is the sum of $m$ such random variables should follow a Lévy distribution with a norming constant $\sim m^{1 /(\lambda-1)}$, so long as $R_{m}$ is less than the correlation length $\xi$. In other words, the length $R_{m}$ of a segment which contains $m$ probes scales as $m^{1 / \lambda-1)}$. This is valid up to $R_{m} \sim \xi$ but fails as $R_{m}$ increases beyond that. $\xi$ is the same correlation length that appears in Eq. (3) for the noninteracting case. Let $m_{>}$be the number of STPs in a segment of length $\xi$. Then $m_{>} \sim \xi^{\lambda-1}$. Hence in a system of length $L$, the total number of probes $N_{0}$ can be written as $N_{0}=(L / \xi) \xi^{\lambda-1}$, which implies that the correlation length $\xi \sim \rho_{0}^{-1 /(2-\lambda)}$ and hence $\tau \sim \xi^{z_{0}} \sim \rho_{0}^{-z_{0} /(2-\lambda)}$, where $z_{0}$ is the dynamical critical exponent of the system.

We have monitored the dynamical correlation functions $C_{0}(t), B_{0}(t)$, and $\Delta(t)$, as defined in Eqs. (5), (8), and (10), respectively. Our numerical simulations indicate that these quantities follow the same scaling form as in the noninteracting case $\epsilon=0[6]$. Moreover they continue to show cross-

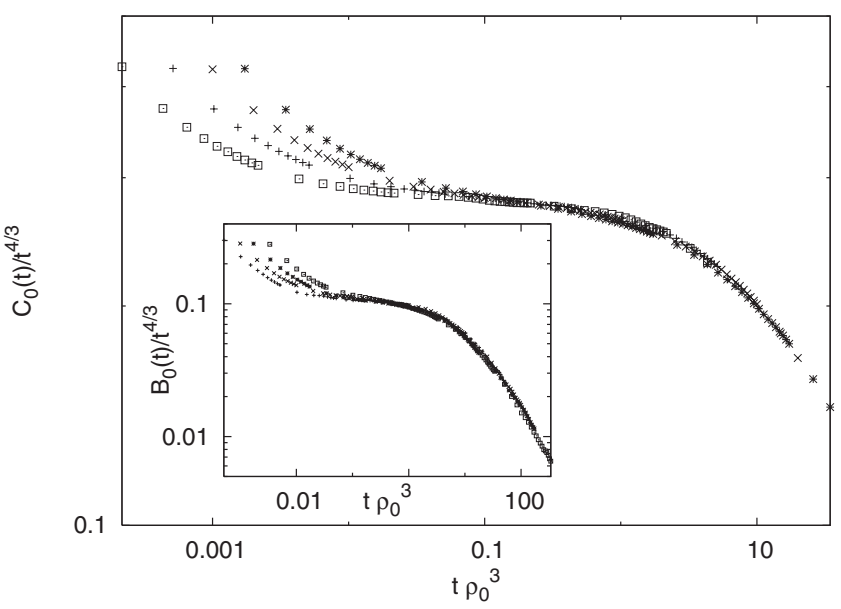

FIG. 6. Scaling collapse for $C_{0}(t)$ for $\epsilon=0.2$ and $\rho_{0}$ $=0.06,0.08,0.1,0.12$. The inset shows scaling collapse for $B(t)$ with $\epsilon=0.2$ and $\rho_{0}=0.08,0.1,0.12,0.15$. We have used $L$ $=16384$.

over at a time scale $\tau \sim \rho_{0}^{-3}$, very similar to the $\epsilon=0$ case. In Fig. 6 we show the scaling collapse for $C_{0}(t)$ and $B_{0}(t)$. We present our data for $\Delta(t)$ in Fig. 7.

In the case of two probes, one might expect $\Delta(t)$ would show the same scaling behavior as the second moment of the distribution $P(r, t)$ in Eq. (15), i.e., $\Delta(t)$ should grow with time as $t^{(3-b) / z}$. But our numerical simulations show that irrespective of the value of $\epsilon, \Delta(t)$ always grows linearly with time (as with $\epsilon=0$ ). We have shown our results for $\epsilon=0.5$ in the inset in Fig. 7 .

Note that the above scaling analysis and our numerical simulation presented in Figs. 6 and 7 point toward $z_{0} /(2$ $-\lambda)=3$. If $\lambda=b(\epsilon)$ as reported in Ref. [12], then for larger values of $\epsilon$ this would lead to $z_{0}$ smaller than unity. For example, for $\epsilon=0.5$, we have verified that the above scaling form remains valid (see Fig. 7), which would imply $z_{0}$ $\simeq 0.54$ if $\lambda=b(\epsilon)$.

The other (simpler) alternative is that $z_{0}=z=3 / 2$ and $\lambda$ $=3 / 2$ as in the $\epsilon=0$ case. This scenario would explain the

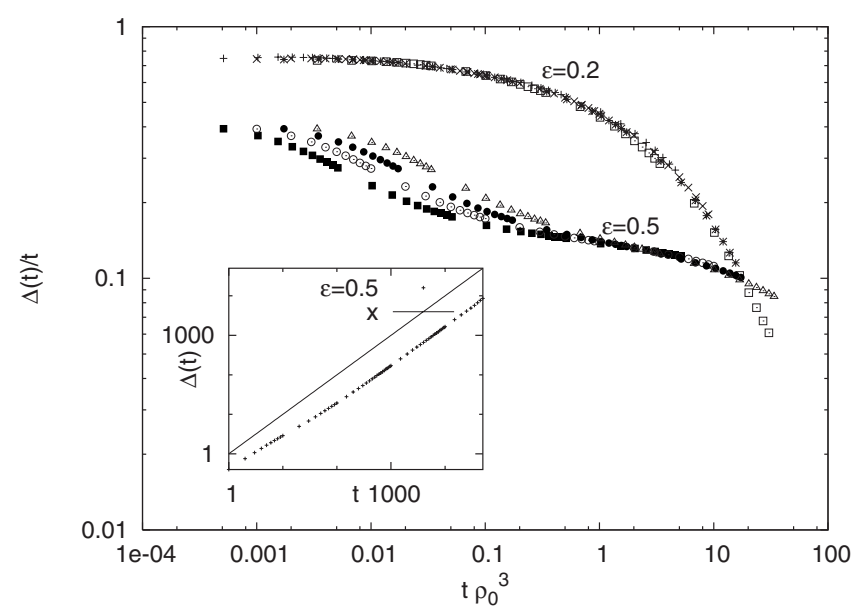

FIG. 7. Scaling collapse for $\Delta(t)$ for finite $\epsilon$ values. We have used $\rho_{0}=0.08,0.1,0.12,0.15$ and $L=16384$. The inset shows the linear growth of $\Delta(t)$ for $L=16384$ in the presence of two probes. 
observed $\rho_{0}$ dependence of crossover time $\tau$. In the case of two probes, the above value of $\lambda$ is consistent with the large time growth exponent of the average separation $\langle r(t)\rangle$ between the probe pair (shown in Fig. 4) and also with the linear growth of $\Delta(t)$ shown in the inset of Fig. 7 .

\section{CONCLUSION}

In this paper, we have studied the dynamics of shocktracking probe particles in a one-dimensional KLS model of driven particles with nearest neighbor Ising interaction $\epsilon$. In particular, we have examined our results in the light of two different theoretical scenarios. The first scenario is based on an approximate mapping of the problem to a zero-range process, and leads to the conclusion that critical exponents characterizing power-law decays depend continuously on the strength of interaction $\epsilon$. The second scenario is based on the premise that since the correlations induced by Ising interactions are short-ranged, asymptotic scaling properties which involve large distances and large times should be independent of $\epsilon$, and the same as at $\epsilon=0$. The results of our numerical studies on the dynamical properties of the probe particles lend support to the second scenario.

We find that in presence of only two probe particles in the system, starting from a steady state configuration where the two probes were nearest neighbors, the average distance $\langle r(t)\rangle$ between them shows a crossover in time. For an initial time regime $\langle r(t)\rangle$ the growth is consistent with a power law with an $\epsilon$-dependent exponent $[2-b(\epsilon)] / z[12,13]$. However, for large enough time, the growth occurs with an exponent $\simeq 1 / 3$, the value expected for a noninteracting medium, consistent with the second scenario discussed above. In addition, our study of the cumulative distribution of the probeseparation shows that for large time, the distribution function does not follow an $\epsilon$-dependent scaling form as claimed in Ref. [13] but can be described by a form expected for $\epsilon=0$, which again supports the second scenario mentioned above.

For a small but finite density of the probes, the dynamical correlation functions show a similar scaling form as for $\epsilon$ $=0$ [6]. These scaling forms involve a crossover time scale $\tau$ that diverges for small $\rho_{0}$ as $\rho_{0}^{-3}$, as found for the noninteracting case [6]. We have seen that an $\epsilon$-dependent exponent $b(\epsilon)$ would lead to an $\epsilon$-dependent dynamical exponent $z_{0}$ which may even become less than unity for larger values of $\epsilon$. The other option, an $\epsilon$-independent dynamical exponent $z_{0}=3 / 2$, is consistent with the second scenario outlined above, according to which, turning on a short-ranged Ising interaction in the medium does not change the large time and large distance properties of the system.

It is not yet completely clear why the KLS-ZRP mapping does not seem to yield results which agree with the numerical results. One possible reason is that the ZRP mapping does not take into account the non-Markovian movement of the probes. This lack of agreement also opens up the question of the nature of the complete phase diagram for the problem under study, including negative values of $\epsilon$.

\section{ACKNOWLEDGMENT}

We acknowledge useful discussions with G. M. Schütz, D. Mukamel, Y. Kafri, A. Rakos, M. R. Evans, and D. Dhar.
[1] D. Weihs, T. G. Mason, and M. A. Teitell, Biophys. J. 91, 4296 (2006).

[2] H. Oikawa and H. Nakanishi, J. Chem. Phys. 115, 3785 (2001).

[3] G. V. Soni, B. M. Jaffar Ali, Y. Hatwalne, and G. V. Shivashankar, Biophys. J. 84, 2634 (2003).

[4] P. Habdas, D. Schaar, A. C. Levitt, and E. R. Weeks, Europhys. Lett. 67, 477 (2004).

[5] Y. Kafri, E. Levine, D. Mukamel, G.M. Schütz and R.D. Willmann, Phys. Rev. E 68, 035101(R) (2003).

[6] S. Chatterjee and M. Barma, J. Stat. Mech.: Theory Exp. 2007, L01004 (2007).

[7] T. M. Liggett, Interacting Particle Systems (Springer, Berlin, 1985).

[8] P. A. Ferrari, C. Kipnis, and S. Saada, Ann. Probab. 19, 226 (1991).

[9] B. Derrida, S. A. Janowsky, J. L. Lebowitz, and E. R. Speer, J. Stat. Phys. 73, 813 (1993).

[10] S. Katz, J. L. Lebowitz, and H. Spohn, J. Stat. Phys. 34, 497 (1984).

[11] J. M. Luck and C. Godrèche, J. Stat. Mech.: Theory Exp. (2006), 08009.

[12] E. Levine, D. Mukamel, and G. M. Schütz, Europhys. Lett. 70, 565 (2005).
[13] A. Rakos, E. Levine, D. Mukamel, and G. M. Schütz, J. Stat. Mech.: Theory Exp. (2006), 11001.

[14] P. A. Ferrari and L. R. G. Fontes, Probab. Theory Relat. Fields 99, 305 (1994).

[15] B. Derrida and M. R. Evans, J. Phys. A 32, 4833 (1999).

[16] C. Boutillier, P. François, K. Mallick, and S. Mallick, J. Phys. A 35, 9703 (2002).

[17] H. Beijeren, J. Stat. Phys. 63, 47 (1991).

[18] S. Gupta, S. N. Majumdar, C. Godrèche, and M. Barma, Phys. Rev. E 76, 021112 (2007).

[19] M. R. Evans, Braz. J. Phys. 30, 42 (2000).

[20] Y. Kafri, E. Levine, D. Mukamel, and J. Török, J. Phys. A 35, L459 (2002).

[21] M. R. Evans, E. Levine, P. K. Mohanty, and D. Mukamel, Eur. Phys. J. B 41, 223 (2004).

[22] Throughout this paper, we have considered probe particles embedded in a KLS ring and found a large time crossover in the probe dynamics as discussed above. Instead of a KLS model, when the probe particles are embedded in a medium, such that certain movements of the probes become temporally uncorrelated (see Ref. [13] for details), then no such crossover effects have been observed. In this case of "mixed dynamics" of the probe particles, an $\epsilon$-dependent scaling form seems to remain valid, within the range of time we have investigated. 